\title{
THE DEAD LEAVES MODEL: A GENERAL TESSELLATION MODELING OCCLUSION
}

\author{
CHARLES BORDENAVE, ${ }^{*}$ Ecole Normale Supérieure and INRIA \\ YANN GOUSSEAU ${ }^{* * * * *}$ AND \\ FRANÇOIS ROUEFF, ${ }^{* * * * * *}$ Télécom Paris
}

\begin{abstract}
In this article, we study a particular example of general random tessellation, the dead leaves model. This model, first studied by the mathematical morphology school, is defined as a sequential superimposition of random closed sets, and provides the natural tool to study the occlusion phenomenon, an essential ingredient in the formation of visual images. We generalize certain results of G. Matheron and, in particular, compute the probability of $n$ compact sets being included in visible parts. This result characterizes the distribution of the boundary of the dead leaves tessellation.
\end{abstract}

Keywords: General tessellation; dead leaves model; typical cell; image modeling

2000 Mathematics Subject Classification: Primary 60D05

Secondary 60G55; 52A22; 68U10

\section{Introduction}

The dead leaves model was introduced by Matheron in [18], and results from the sequential superimposition of random sets. As such, it provides the natural tool for studying nonlinear occlusion phenomena, which are of great importance in image modeling and processing. However, to the best of the authors' knowledge, this model has not been systematically investigated, and even its definition lacks some precision. Our purpose in this paper is twofold: first, to provide a rigorous definition of the model as a random tessellation; second, to give new proofs or extensions of Matheron's results in the framework of Palm calculus.

A first motivation to study this model comes from applications. Among existing stochastic models for natural images, the dead leaves model is the only one whose definition agrees with their physical formation. Several recent studies have demonstrated the ability of specific dead leaves models to reproduce most known statistics of natural images; see [23], [1], and [16]. The model has also been proposed as a tool to resample random fields for texture synthesis; see [10]. Other examples of applications come from materials sciences; see [14] and [8].

As a second motivation, let us stress that the dead leaves model provides nontrivial examples of general random tessellations, in the sense that their cells are general closed sets. In particular, they are not necessarily polygonal, connected, or convex, as is the case for the most popular tessellation models, such as Poisson flats or Voronoi or Delaunay tessellations. Note that

\footnotetext{
Received 22 June 2004; revision received 20 December 2005.

* Postal address: Département d'Informatique, Ecole Normale Supérieure, 45 rue d’Ulm, F-75230 Paris Cedex 05, France. Email address: charles.bordenave@ens.fr

** Postal address: Département Traitement du Signal et des Images, CNRS UMR 5141, ENST, 46 rue Barrault, 75634

Paris Cedex 13, France.

*** Email address: gousseau@tsi.enst.fr

**** Email address: roueff@tsi.enst.fr
} 
nonconvex and nonpolygonal cells are encountered in the case of Johnson-Mehl tessellation (see, e.g. [26, pp. 313, 333]), but that there are relatively few such examples. Therefore, there are few studies of 'general' tessellations, even though classical formulae originally proved in the convex and polygonal case have been shown to hold in more general contexts; see [25], [27], and [6].

In Section 2 we first recall some facts on random closed sets and slightly reformulate notions from [21] and [25] to define random tessellations and typical cell distributions. In Section 3 we define the dead leaves model as a random tessellation obtained from an initial Poisson process, and give some of its elementary properties. Then, in Section 4, we generalize the results of Matheron. In order to do so in a rigorous way, we make use of point process theory through the systematic use of Palm calculus. We first give the probability of $n$ compact sets being included in $n$ different visible parts, a result that completely characterizes the distribution of the boundary of our model as a random closed set. Then we compute the distribution of 'objects' that remain completely visible. Eventually, we recover, in the Palm calculus framework, a nice result of Matheron giving the length distribution of the intersection of objects with a line of fixed direction, stating in particular that its expectation is divided by two as a result of occlusion.

\subsection{Previous work}

The dead leaves model was introduced in [18], a technical report written in an informal style, yet which contained all the relevant basic ideas. The model was defined as the superimposition of infinitesimal Boolean models, and formulae for the probability of a compact set to be included in a visible part and for the distribution of completely visible parts, among other things, were derived. Most of these definitions and results are stated in the book by Serra [24]. Jeulin further studied this model in [13], still using the same infinitesimal formalism, and gave an explicit formula for the joint probability of two compact sets to be included in visible parts. In [12] he generalized the model to the case of random functions and extended to this setting formulae both for the distribution of visible parts and for inclusion probabilities. Cowan and Tsang, in a very interesting paper [5], made use of mean value formulae for tessellations to derive the expectations of quantities such as the number of connected components of visible parts and the length of their boundaries per surface unit.

\section{Basic definitions}

\subsection{Closed sets and tessellations}

Let $\mathcal{F}, g$, and $\mathcal{K}$ respectively be the sets of all closed, open, and compact sets of $\mathbb{R}^{d}, d \geq 1$. For any $A \subset \mathbb{R}^{d}$, we write

$$
\mathcal{F}^{A}=\{F \in \mathcal{F}: F \cap A=\varnothing\} \quad \text { and } \quad \mathcal{F}_{A}=\{F \in \mathcal{F}: F \cap A \neq \varnothing\} .
$$

The Borel $\sigma$-field, $\mathscr{B}_{\mathcal{F}}$, on $\mathcal{F}$ is generated by the basis of open sets $\left\{\mathcal{F}^{K}, K \in \mathcal{K} ; \mathcal{F}_{G}, G \in \mathcal{G}\right\}$. Borel sets are defined on $\mathscr{g}$ and $\mathcal{K}$ in a way similar to those of $\mathcal{F}$; see [19, p. 11]. A random closed set of $\mathbb{R}^{d}$ is a measurable function from a probability space $(\Omega, \&, \mathrm{P})$ into $\left(\mathcal{F}, \mathscr{B}_{\mathcal{F}}\right)$. For any sets $A$ and $B$, we write

$$
A \ominus B=\left\{x \in \mathbb{R}^{d}: x+\check{B} \subset A\right\} \quad \text { and } \quad A \oplus B=\{x+y: x \in A, y \in B\},
$$

where $\check{B}=\{-x, x \in B\}$. The set $A \ominus \check{B}$ is called the erosion of $A$ by $B$, and $A \oplus \check{B}$ the dilation of $A$ by $B$. Measurability properties of the operators ' $\ominus$ ' and ' $\oplus$ ' were established in [19, pp. 19-20]. 
A $\sigma$-finite measure on $\mathcal{F}^{\prime}:=\mathscr{F} \backslash\{\varnothing\}$ (endowed with its Borel $\sigma$-algebra, $\mathscr{B}_{\mathcal{F}^{\prime}}$ ) is a measure taking finite values on $\mathcal{F}_{K}$ for all $K \in \mathcal{K}$; see [19, p. 57]. We denote by $\mathcal{N}_{\mathcal{F}^{\prime}}$ the set of $\sigma$-finite counting measures on $\left(\mathcal{F}^{\prime}, \mathcal{B}_{\mathcal{F}^{\prime}}\right)$. For all $M \in \mathcal{N}_{\mathcal{F}^{\prime}}$, we write $M=\sum_{i} \delta_{F_{i}}$, where $\delta_{F_{i}}$ is the unit-mass measure at point $F_{i}$. The boundary of $M$ is defined as $\partial M=\bigcup_{i} \partial F_{i}$, where $\partial F_{i}$ denotes the topological boundary of $F_{i}$. A point process on $\mathcal{F}^{\prime}$ is a measurable function from a probabilistic space to $\left(\mathcal{N}_{\mathcal{F}^{\prime}}, \mathscr{B}_{\mathcal{N}_{\mathcal{F}^{\prime}}}\right)$, where $\mathscr{B}_{\mathcal{N}_{\mathcal{F}^{\prime}}}$ is the usual $\sigma$-field on $\mathcal{N}_{\mathcal{F}^{\prime}}$; see, e.g. [7, Chapter 6].

Following Stoyan [25], a tessellation of $\mathbb{R}^{d}$ is defined as follows.

Definition 1. Let $T=\sum_{i} \delta_{F_{i}} \in \mathcal{N}_{\mathcal{F}^{\prime}}$. We say that $T$ is a tessellation of $\mathbb{R}^{d}$ if

(i) $\bigcup_{i} F_{i}=\mathbb{R}^{d}$ and,

(ii) for all $i \neq j$, Int $F_{i} \cap F_{j}=\varnothing$, where Int $F$ denotes the interior of $F$,

or, equivalently, if $\left\{\left(\operatorname{Int} F_{i}\right)_{i}, \partial T\right\}$ is a partition of $\mathbb{R}^{d}$.

Note that $T \in \mathcal{N}_{\mathcal{F}^{\prime}}$ implies that the number of cells $F_{i}$ hitting a compact set is finite. This condition was added in the original definition in [25], where the $F_{i}$ are marks of a point process, $N=\sum_{i} \delta_{x_{i}}$, on $\mathbb{R}^{d}$ (where $x_{i}$ is called the centroid of $F_{i}$ ). The centroids are unimportant in the definition of a tessellation, but they are quite useful in defining the typical cell distribution, as we will recall below.

Let $\mathcal{T}$ be the set of all tessellations in $\mathcal{N}_{\mathcal{F}^{\prime}}$. By expressing assertions (i) and (ii) of Definition 1 as limits of the elementary set operations $\left(F, F^{\prime}\right) \mapsto F \cup F^{\prime},\left(F, F^{\prime}\right) \rightarrow F \cap F^{\prime}$, and $F \mapsto \partial F$, which are measurable as a consequence of [19, Sections 1-2], we easily find that $\mathcal{T} \in \mathcal{B}_{\mathcal{N}_{\mathcal{F}^{\prime}}}$. A random tessellation of $\mathbb{R}^{d}$ is then defined as a point process, $T$, on $\mathcal{F}^{\prime}$, such that $T \in \mathcal{T}$ almost surely (a.s.). Classical examples of random tessellations (see the references in [26, Chapter 10] and [22]) include Poisson hyperplane processes and Delaunay, Voronoi, and Johnson-Mehl tessellations. A standard approach (see, e.g. [2], [4], [20], [21], or [26, Chapter 10]), which applies in these examples, is to define $\partial T$ directly as a random closed set without considering the underlying random tessellation. However, it is not always possible to recover the $F_{i}$ from $\partial T$ (as they may not be connected; see [6] and Remark 2, below, for a precise example).

\subsection{Typical cell distribution}

In [21] a typical cell was defined using the Palm distribution of a simple marked point process, $N=\sum_{i} \delta_{x_{i}, F_{i}}$, of points in $\mathbb{R}^{n}$ with marks in $\mathcal{F}^{\prime}$ that is stationary with respect to shifts $N \mapsto \sum \delta_{x_{i}-x, F_{i}-x}, x \in \mathbb{R}^{d}$. More precisely, let us denote by $\mu$ the intensity of $N$, which we assume to be finite, and by $\mathbb{P}_{N}^{0}$ its Palm distribution. Let $x_{0}$ be the point nearest to the origin and $F_{0}$ its corresponding cell. Then the typical cell distribution is defined on the $\sigma$-field, $\mathcal{I}$, of all translation-invariant events in $\mathcal{B}_{\mathcal{F}^{\prime}}$ by $\chi \mapsto \mathbb{P}_{N}^{0}\left(F_{0} \in \chi\right), \chi \in \mathcal{I}$. A result of [21], proved for tessellations whose cells are bounded polytopes, can easily be extended as follows.

Proposition 1. Let B be a Borel set in $\mathbb{R}^{d}$ such that

$$
0<v\left(F_{i} \oplus B\right)<\infty \text { for all i, a.s., }
$$

where $v$ is the Lebesgue measure on $\mathbb{R}^{n}$. Then $\mu=\mathbb{E}\left[\sum_{i} \mathbf{1}\left(0 \in F_{i} \oplus B\right) / v\left(F_{i} \oplus B\right)\right]$ and

$$
\mathbb{P}_{N}^{0}\left(F_{0} \in \chi\right)=\frac{1}{\mu} \mathbb{E}\left[\sum_{i} \frac{\mathbf{1}\left(0 \in F_{i} \oplus B\right) \mathbf{1}\left(F_{i} \in \chi\right)}{\nu\left(F_{i} \oplus B\right)}\right], \quad \chi \in \mathcal{L} .
$$


When starting from a stationary point process, $M=\sum_{i} \delta_{F_{i}}$, on $\mathcal{F}^{\prime}$, a marked point process, $N$, can be obtained by constructing points $x_{i}=\Delta\left(F_{i}\right)$, where $\Delta$ is such that $\Delta\left(F_{i}-x\right)=$ $\Delta\left(F_{i}\right)-x$. Classical examples for $\Delta$ include the set-centroid, the median point, and the extremal point in a given direction. Observe that, under condition (1), it is always possible to define such a set-centroid by taking for each coordinate the median of the marginal measure of $v$ restricted to $F_{i} \oplus B$; for instance, the first coordinate is then defined as the smallest $x$ such that $v\left(\left(F_{i} \oplus B\right) \cap(-\infty, x] \times \mathbb{R}^{d-1}\right) \geq v\left(F_{i} \oplus B\right) / 2$. As was noticed in [21], the typical cell distribution should not depend on the choice of $x_{i}$, which is ensured by Proposition 1, provided that a Borel set $B$ can be found for which (1) is fulfilled. This will be the case for the dead leaves model considered below.

In order to define the typical cell of a tessellation, assume that

$$
0<v\left(F_{i}\right)<\infty \text { and } v\left(\partial F_{i}\right)=0, \quad \text { for all } i, \text { a.s. }
$$

Note that the first of these conditions is (1) with $B=\{0\}$. The second condition enables us to define $F_{\{x\}}$ almost everywhere as the cell to which the point $x$ belongs. By the stationarity of $N, F_{\{0\}}$ is a.s. defined. Applying Proposition 1 then yields

$$
\mu=\mathbb{E}\left[\frac{1}{v\left(F_{\{0\}}\right)}\right] \quad \text { and } \quad \mathbb{P}_{N}^{0}\left(F_{0} \in \chi\right):=\frac{1}{\mu} \mathbb{E}\left[\frac{1\left(F_{\{0\}} \in \chi\right)}{v\left(F_{\{0\}}\right)}\right], \quad \chi \in \mathcal{L} .
$$

We thus obtain the formula of the typical cell distribution derived in [20], [21] (when the $F_{i}$ are bounded polytopes), and [4] (when the $F_{i}$ are uniformly bounded polytopes).

We end this section with a limit theorem. Let $B_{n}=B\left(0, r_{n}\right)$ be the ball of radius $r_{n}$, $r_{n} \rightarrow \infty$, centered at 0 . Let $\left(A_{n}\right)_{n \in \mathbb{N}}$ be any increasing sequence of compact convex sets such that, for all $n, B_{n} \subset A_{n}$. The individual ergodic theorem (Proposition 10.2.II of [7]) easily yields the following result.

Proposition 2. If $N$ is ergodic and satisfies (2), then, for all $\chi \in \mathcal{L}$,

$$
\lim _{n} \frac{\sum_{i} \mathbf{1}\left(F_{i} \in \chi\right) v\left(F_{i} \cap A_{n}\right) / v\left(F_{i}\right)}{\sum_{i} v\left(F_{i} \cap A_{n}\right) / v\left(F_{i}\right)}=\mathbb{P}_{N}^{0}\left(F_{0} \in \chi\right) \quad \text { a.s. }
$$

Equation (3) is a weighted average in which each $F_{i}$ has a weight equal to its proportion included in $A_{n}$. From a statistical point of view, (3) can be used in deriving a strongly consistent estimator of $\mathbb{P}_{N}^{0}\left(F_{0} \in \chi\right)$, for a given $\chi \in \mathcal{I}$. Under a stronger hypothesis on the cells, there may be different sequences having the same limit as in (3). For example, if the cells are uniformly bounded (as in [4]), (3) implies that

$$
\mathbb{P}_{N}^{0}\left(F_{0} \in \chi\right)=\lim _{n} \frac{\sum_{i} \mathbf{1}\left(F_{i} \in \chi\right) \mathbf{1}\left(F_{i} \subset A_{n}\right)}{\sum_{i} \mathbf{1}\left(F_{i} \subset A_{n}\right)}=\lim _{n} \frac{\sum_{i} \mathbf{1}\left(F_{i} \in \chi\right) \mathbf{1}\left(F_{i} \cap A_{n} \neq \varnothing\right)}{\sum_{i} \mathbf{1}\left(F_{i} \cap A_{n} \neq \varnothing\right)} \quad \text { a.s. }
$$

Sufficient conditions under which these equalities hold were studied in [6].

\section{The dead leaves model}

\subsection{Definition}

The dead leaves model is obtained through sequential superimposition of random objects 'falling' on $\mathbb{R}^{d}$. Let $\sum_{i \in \mathbb{N}} \delta_{x_{i}, t_{i}}$ be a homogeneous Poisson point process on the half-space $\mathbb{R}^{d} \times(-\infty, 0]$, with intensity 1 . Let $\mathrm{P}$ be a probability measure on $\left(\mathcal{F}, \mathscr{B}_{\mathcal{F}}\right)$, and let $\left(X_{i}\right)_{i \in \mathbb{N}}$ 
be independent, identically distributed random variables on $\mathcal{F}$ that have distribution $\mathrm{P}$ and are independent of the Poisson point process above. Equivalently, $\Phi=\sum_{i} \delta_{x_{i}, t_{i}, X_{i}}$ is a Poisson point process on $\mathbb{R}^{d} \times(-\infty, 0] \times \mathcal{F}$ with intensity measure $v(\mathrm{~d} x) \mathrm{d} t \mathrm{P}(\mathrm{d} X)$.

We write $(\Omega, \&, \mathbb{P})$ for the probabilistic space on which $\Phi$ is defined and $\mathbb{E}$ for the expectation with respect to $\mathbb{P}$. From now on, $X$ will always denote a random variable on $\mathcal{F}$ that has distribution $\mathrm{P}$ and is independent of all other variables, and $\mathrm{E}$ will denote the expectation with respect to $\mathrm{P}$.

Definition 2. For all $i \in \mathbb{N}$, the random closed set $x_{i}+X_{i}$ is called a leaf and

$$
V_{i}=\left(x_{i}+X_{i}\right) \backslash \bigcup_{t_{j} \in\left(t_{i}, 0\right)}\left(x_{j}+\operatorname{Int} X_{j}\right)
$$

is called a visible part.

From now on we assume that $X$ satisfies the following three conditions.

(C1) For all $K \in \mathcal{K}, \mathrm{E}[v(X \oplus K)]<\infty$.

(C2) There exists a ball, $B$, with strictly positive radius, such that $\mathrm{E}[v(X \ominus B)]>0$.

(C3) $X$ is a regular closed set, i.e. $X$ is the closure of its interior, P-a.s.

Proposition 3. We denote by $M$ the point process on $\mathcal{F}^{\prime}$ obtained by removing from the collection $\left\{V_{i}\right\}$ all sets with empty interiors, that is,

$$
M=\sum_{i} \mathbf{1}\left\{\operatorname{Int} V_{i} \neq \varnothing\right\} \delta_{V_{i}} .
$$

Then $M$ is a random tessellation of $\mathbb{R}^{d}$. Moreover, $N=\sum_{i} \mathbf{1}\left(\right.$ Int $\left.V_{i} \neq \varnothing\right) \delta_{x_{i}, V_{i}}$ is stationary, mixing, and has finite intensity.

Remark 1. The condition Int $V_{i} \neq \varnothing$ in the definitions of $M$ and $N$ is adopted for convenience as it eliminates visible parts with zero $d$-dimensional Lebesgue measures. The question arises as to whether $M^{\prime}:=\sum_{i} \mathbf{1}\left(V_{i} \neq \varnothing\right) \delta_{V_{i}}$ also has such a property. For simple examples of $X$, it is easily shown that $M=M^{\prime}$ a.s. but we do not know whether this equality is true under the general assumptions (C1)-(C3). In any case, because (4) implies that $\partial V_{i} \subset \bigcup_{t_{j}>t_{i}} \partial\left\{\right.$ Int $\left.V_{j}\right\}$, we always have $\partial M=\partial M^{\prime}$.

In order to prove Proposition 3 we will make use of the following two lemmas. The first one, which is easy to prove by referring to the definition of the intensity of the Poisson point process $\Phi$, will be needed repeatedly in the sequel.

Lemma 1. Let $K$ be a bounded Borel set, let $s_{1}$ and $s_{2}$ satisfy $-\infty<s_{1}<s_{2}<0$, and define

$$
\begin{aligned}
& \Phi_{K}\left(s_{1}, s_{2}\right):=\sum_{i} \mathbf{1}\left(t_{i} \subset\left(s_{1}, s_{2}\right] \text { and } K \subset x_{i}+X_{i}\right), \\
& \Phi^{K}\left(s_{1}, s_{2}\right):=\sum_{i} \mathbf{1}\left(t_{i} \subset\left(s_{1}, s_{2}\right] \text { and } K \cap x_{i}+X_{i} \neq \varnothing\right) .
\end{aligned}
$$

These functions are Poisson random variables with respective means $\left(t_{2}-t_{1}\right) \mathrm{E}[v(X \ominus \check{K})]$ and $\left(t_{2}-t_{1}\right) \mathrm{E}[v(X \oplus \check{K})]$. 
Lemma 2. If $K$ is a Borel set of $\mathbb{R}^{d}$ such that $\mathrm{E}[v(X \ominus \check{K})]>0$, then $K$ is almost surely covered by some leaf $x_{i}+X_{i}$. As a consequence, any bounded set is a.s. covered by a finite number of leaves.

Proof. Let us fix $t<0$. By Lemma 1, the probability, $\mathbb{P}\left(\Phi_{K}(t, 0)=0\right)$, that none of the leaves $x_{i}+X_{i}$, with $t<t_{i}<0$, satisfies $K \subset x_{i}+X_{i}$ is $\exp (t \mathrm{E}[v(X \ominus \check{K})])$, which yields the first assertion. Now let $B$ be a ball such that (C2) is satisfied, that is, $\mathrm{E}[v(X \ominus B)]>0$. Since any bounded set $K$ is covered by a finite number of balls with the same radius as $B$, it follows that $K$ is also covered by $\bigcup_{t_{i}>T}\left(x_{i}+X_{i}\right)$ for some $T<0$.

Proof of Proposition 3. Let us now show that $M \in \mathcal{N}_{\mathcal{F}}, \mathbb{P}$-a.s. In fact, we will show that

$$
M^{\prime}:=\sum_{i} \mathbf{1}\left(V_{i} \neq \varnothing\right) \delta_{V_{i}} \in \mathcal{N}_{\mathcal{F}^{\prime}} \quad \mathbb{P} \text {-a.s. }
$$

(which implies that $M \in \mathcal{N}_{\mathcal{F}^{\prime}}$ ), that is, that only a finite number of visible parts $V_{i}$ may intersect a given compact set $K$. By Lemma 2, there $\mathbb{P}$-a.s. exists a negative $T$ such that $K$ is covered by leaves, $x_{i}+X_{i}$, satisfying $t_{i}>T$. It follows that the visible parts intersecting $K$ correspond to leaves falling after time $T$. The number of such leaves is thus $\Phi^{K}(T, 0)$, which is $\mathbb{P}$-a.s. finite, by Lemma 1 under condition (C2). To show that $M$ is a random tessellation, we now verify that it satisfies conditions (i) and (ii) of Definition 1. Let $T<0$. Since $\bigcup_{t_{i}>T} V_{i} \subseteq \bigcup_{t_{i}>T}\left(x_{i}+X_{i}\right)$ and since a point in $x_{i}+X_{i}$ either belongs to $V_{i}$ or to $x_{j}+\operatorname{Int} X_{j}$ for some $t_{j}>t_{i}$, we have $\bigcup_{t_{i}>T}\left(x_{i}+X_{i}\right)=\bigcup_{t_{i}>T} V_{i}$. Therefore by Lemma 2 we have $\bigcup_{i} V_{i}=\mathbb{R}^{d} \mathbb{P}$-a.s. We observe from (C3) that Int $V_{i}=\left(x_{i}+\operatorname{Int} X_{i}\right) \cap \bigcap_{t_{j}>t_{i}}\left(x_{j}+X_{j}\right)^{\mathrm{c}}$. It follows that Int $V_{i}=\varnothing$ if and only if $V_{i} \subset \bigcup_{t_{j}>t_{i}}\left(x_{j}+X_{j}\right)=\bigcup_{t_{j}>t_{i}} V_{j}$. Indeed, the 'if' part is obvious, while the 'only if' part is obtained by observing that $\left(x_{i}+\operatorname{Int} X_{i}\right) \subseteq \bigcap_{t_{j}>t_{i}}\left(x_{j}+X_{j}\right)$ implies the same inclusion for $\overline{x_{i}+\text { Int } X_{i}}=x_{i}+X_{i} \supseteq V_{i}$.

Finally, consider a realization of $\Phi$ such that $M^{\prime} \in \mathcal{N}_{\mathcal{F}^{\prime}}$ and $\bigcup_{i} V_{i}=\mathbb{R}^{d}$, which is $\mathbb{P}$ a.s. true, as we have shown above. Pick any point $x \in \mathbb{R}^{d}$. Since $M^{\prime} \in \mathcal{N}_{\mathcal{F}^{\prime}}$, there exists a positive, finite number of indices $i$ such that $x \in V_{i}$, and hence one $i$ such that $x \in V_{i}$ and $x \notin V_{j}$ for all $t_{j}>t_{i}$. By the above characterization, this implies that Int $V_{i} \neq \varnothing$. Hence,

$$
\bigcup_{i}\left\{V_{i}: \text { Int } V_{i} \neq \varnothing\right\}=\mathbb{R}^{d}
$$

that is, $M$ satisfies condition (i) of Definition 1. Condition (ii) of Definition 1 is easily obtained from (4) and (C3) by considering the cases $t_{j}>t_{i}$ and $t_{i}>t_{j}$ successively.

Next we demonstrate stationarity and the mixing property. Define

$$
\Pi: \sum_{i} \delta_{x_{i}, t_{i}, X_{i}} \mapsto \sum_{i} 1\left(\operatorname{Int} V_{i} \neq \varnothing\right) \delta_{x_{i}, V_{i}} .
$$

Recall that $\mathbb{P}$ denotes the distribution of the initial (homogeneous) Poisson point process $\Phi$, meaning that $\mathbb{P}_{\Pi}:=\mathbb{P} \circ \Pi^{-1}$ is the distribution of $N$. Furthermore, observe that translations of the $x_{i}$ correspond to translations of the $V_{i}$ through $\Pi$. It follows that the stationarity and the mixing property of $N$ (with respect to shifts $N \mapsto \sum_{i} \delta_{x_{i}-x, V_{i}-x}, x \in \mathbb{R}^{d}$ ) are inherited from $\Phi$.

It remains to prove that the intensity, $\mu$, of $N$ is finite. For all $T<0$, let $N_{T}:=$ $\sum \delta_{x_{i}, V_{i}} \mathbf{1}\left(t_{i}>T\right.$, Int $\left.V_{i} \neq \varnothing\right)$. Let $\mu_{T}$ denote the intensity of $N_{T}$; since

$$
\mu_{T} \leq \mathbb{E}\left[\sum_{i} \mathbf{1}\left(x_{i} \in[0,1]^{n}, t_{i}>T\right)\right] \leq-T,
$$


$\mu_{T}$ is finite. By monotone convergence, since $\mu_{T}$ is nondecreasing as $T$ decreases to $-\infty$, we have $\mu=\lim _{T \rightarrow-\infty} \mu_{T}$. Below we provide a uniform upper bound for $\mu_{T}$, which will thus apply to $\mu$ and conclude the proof. Using Proposition 1 with $B$ given by (C2), we obtain

$$
\begin{aligned}
\mu_{T} & =\mathbb{E}\left[\sum_{i} \frac{\mathbf{1}\left(0 \in V_{i} \oplus B\right)}{v\left(V_{i} \oplus B\right)} \mathbf{1}\left(t_{i}>T, \text { Int } V_{i} \neq \varnothing\right)\right] \\
& \leq v(B)^{-1} \mathbb{E}\left[\sum_{i} \mathbf{1}\left(0 \in x_{i}+X_{i} \oplus B, 0 \notin \bigcup_{t_{i}>t}\left(x_{i}+\operatorname{Int} X_{i} \ominus B\right)\right)\right],
\end{aligned}
$$

where the inequality follows from both $v\left(V_{i} \oplus B\right) \geq v(B)$ and

$$
V_{i} \oplus B \subset\left(x_{i}+X_{i} \oplus B\right) \backslash \bigcup_{t_{i}>t}\left(x_{i}+\operatorname{Int} X_{i} \ominus B\right),
$$

the latter of which in turn follows from (4) and standard properties of morphological operations. Now, Campbell's formula and Slivnyak's theorem (see, e.g. [26, pp. 124, 125]) yield

$$
\mu_{T} \leq \frac{1}{v(B)} \int_{[T, 0] \times \mathbb{R}^{d} \times \mathcal{F}} \mathbf{1}(0 \in x+X \oplus B) \mathbb{P}\left(0 \notin \bigcup_{t_{i}>t}\left(x_{i}+\operatorname{Int} X_{i} \ominus B\right)\right) \mathrm{d} t v(\mathrm{~d} x) \mathrm{P}(\mathrm{d} X) .
$$

Noticing that $\bigcup_{t_{i}>t}\left(x_{i}+\operatorname{Int} X_{i} \ominus B\right)$ is a Boolean model with intensity $t$, we thus obtain

$$
\mu_{T} \leq \frac{1}{v(B)} \mathrm{E}[v(X \oplus B)] \int_{T}^{0} \exp (t \mathrm{E}[v(X \ominus B)]) \mathrm{d} t \leq \frac{1}{v(B)} \frac{\mathrm{E}[v(X \oplus B)]}{\mathrm{E}[v(X \ominus B)]},
$$

which is finite under (C1) and (C2).

In the definition of $M$, we assume that $\sum_{i} \delta_{x_{i}, t_{i}}$ has intensity 1 . However, rescaling the $x_{i}$ is equivalent, up to a global rescaling of the model, to a rescaling of $X$, and any order-preserving modification of the $t_{i}$ is unimportant, as seen from the definition.

Definition 3. The point process $M$, defined in Proposition 3, is called the dead leaves tessellation associated with the random closed set $X$.

Remark 2. The dead leaves model clearly shows the necessity of defining a tessellation through its cells, and not only its boundary. Indeed, visible parts, as defined by (4), are not necessarily connected; see Figure 1.

\subsection{Perfect simulation}

The term 'dead leaves model' originates from a more natural definition that consists in putting each new leaf above the previous ones and then considering the stationary distribution of this Markov process. Let $K$ be a compact set of $\mathbb{R}^{2}$. A classical 'coupling from the past' argument enables perfect simulation of the stationary distribution restricted to $K$, by putting each new leaf below those already fallen, until $K$ is completely covered (see the illustrative web applet by W. S. Kendall at http://www.warwick.ac.uk/statsdpt/staff/WSK/dead.html). This elegant argument was first introduced for the dead leaves model in [15]. In Figures 1 and 2 we show simulations of the model computed this way. To visualize the model, each grain is allocated a random gray level. 

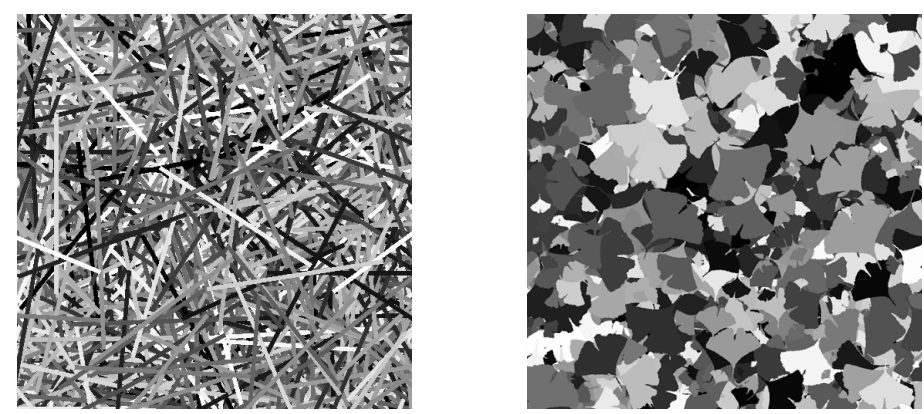

FIGURE 1: Simulations of dead leaves models. Left: the grain $X$ is a rectangle with a direction uniformly distributed in $[0, \pi]$. Right: the grain is a more complicated shape, and its size distribution is uniform.
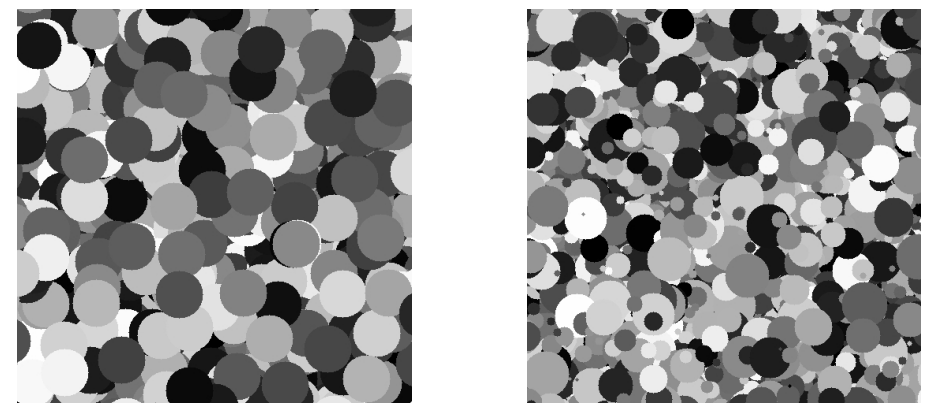

FIGURE 2: Left: simulation of a dead leaves model in which the grain $X$ is a disk with constant radius. Right: simulation of a dead leaves model in which the grain $X$ is a disk with a uniformly distributed radius.

\subsection{Regularity properties of visible parts}

Some almost-sure regularity results about visible parts are a consequence of the following remark. From Lemma 1, a visible part $V_{i}$ is $\mathbb{P}$-a.s. equal to a leaf $x_{i}+X_{i}$ from which a finite number of other leaves have been removed. Now note that if $A$ is a closed set and $B$ is an open set, then $\partial(A \backslash B)=(\partial A \backslash B) \cup(\partial B \cap A)$. It follows that $\partial V_{i}$ is a finite union of sets, each of which is included in $x_{j}+\partial X_{j}$ for some $t_{j} \geq t_{i}$; thus, some regularity properties of $\partial X$ are inherited by the $\partial V_{i}$. Note, however, that the possible convexity of the grain $X$ is not inherited by the $V_{i}$; see Figure 2 .

Proposition 4. We have $v(\partial M)=0 \mathbb{P}$-a.s. if and only if $v(\partial X)=0 \mathrm{P}$-a.s.

Proof. The discussion above implies that $v\left(\partial V_{i}\right) \leq \sum_{t_{j} \geq t_{i}} v\left(\partial X_{i}\right) \mathbb{P}$-a.s. Since $\partial M=$ $\bigcup_{i} \partial V_{i}$, if $v(\partial X)=0 \mathrm{P}$-a.s. then $v(\partial M)=0 \mathbb{P}$-a.s. Now, if $v(\partial M)=0 \mathbb{P}$-a.s. then $v\left(\partial V_{i}\right)=0$ for all $i$; in particular for all cells such that $V_{i}=x_{i}+X_{i}$ (the so-called relief cells, to be studied in Section 4.2). We will see in Remark 4 that this in turn implies that $v(\partial X)=0$ P-a.s.

If Int $V_{i} \neq \varnothing$ then $v\left(V_{i}\right)>0$. Besides, $V_{i} \subset x_{i}+X_{i}$ is $\mathbb{P}$-a.s. bounded, by (C1). If, in addition, $v(\partial X)=0$ P-a.s., then we are in the tessellations framework of Section 2.2. When $v(\partial X)=0$, we say that $X$ is $v$-regular, a property that neither implies nor is implied by (C3). It is easy to find a set $X$ that is $v$-regular but not closed regular, for instance a set containing 
isolated points. To construct a closed regular set that is not $v$-regular, we can proceed as follows (for $d \geq 2$ ). Let $\tilde{v}$ be the $(d-1)$-dimensional Lebesgue measure on the hyperplane $\left\{\mathbf{x}=\left(x_{1}, \ldots, x_{d}\right), x_{1}=\frac{1}{2}\right\}$. Then there exists a homeomorphism, $h:[0,1]^{d} \rightarrow[0,1]^{d}$, such that $v+\tilde{v}=v \circ h$; see [9]. It follows that $X:=h\left(\left[0, \frac{1}{2}\right]^{d}\right)$ is not $v$-regular, although it is closed regular.

\section{Some characteristics of the dead leaves tessellation}

\subsection{Inclusion probabilities and boundary distribution}

The main practical result from the original paper [18] introducing the dead leaves model is concerned with a functional that is defined on compact sets of the plane and equals the probability that a given compact set is included in a visible part of the model. It was shown that, for a non-empty set $K \in \mathcal{K}$,

$$
\mathbb{P}\left(K \subset \text { Int } V_{i} \text { for some } i \in \mathbb{N}\right)=\frac{\mathrm{E}[v(\operatorname{Int} X \ominus \check{K})]}{\mathrm{E}[v(X \oplus \check{K})]} .
$$

The consideration of simple examples for $K$, such as bipoints or segments, leads to valuable geometric information on the model.

In what follows, we generalize this result by studying the probability that $n$ compact sets are included in $n$ distinct visible parts. We define

$$
Q^{(n)}\left(K_{1}, \ldots, K_{n}\right)=\mathbb{P}\left(K_{1} \subset \text { Int } V_{i_{1}}, \ldots, K_{n} \subset \text { Int } V_{i_{n}} \text { for some } t_{i_{1}}<\cdots<t_{i_{n}}<0\right) .
$$

Proposition 5. Let

$$
F^{(n)}\left(K_{1}, \ldots, K_{n}\right)=\mathrm{E}\left[v\left(\operatorname{Int} X \ominus \check{K}_{1}\right)\right] \prod_{j=2}^{n} \mathrm{E}\left[v\left(\left(\operatorname{Int} X \ominus \check{K}_{j}\right) \cap\left(X \oplus \check{\check{K}}_{j-1}\right)^{\mathrm{c}}\right)\right],
$$

and

$$
G^{(n)}\left(K_{1}, \ldots, K_{n}\right)=\prod_{j=1}^{n} \mathrm{E}\left[v\left(X \oplus \underline{\check{K}}_{j}\right)\right]
$$

where, for all $j=1, \ldots, n$,

$$
\underline{K}_{j}=\bigcup_{k=1}^{j} K_{k}
$$

Then

$$
Q^{(n)}\left(K_{1}, \ldots, K_{n}\right)=\frac{F^{(n)}\left(K_{1}, \ldots, K_{n}\right)}{G^{(n)}\left(K_{1}, \ldots, K_{n}\right)} .
$$

Remark 3. Note that (C2) implies $\mathrm{E}[v(X)]>0$ and, thus, that $G^{(n)}\left(K_{1}, \ldots, K_{n}\right)$ does not vanish for non-empty compact sets.

Proof of Proposition 5. Here we choose $n$ fixed, non-empty compact sets, $K_{1}, \ldots, K_{n}$, and write $Q^{(n)}$ for $Q^{(n)}\left(K_{1}, \ldots, K_{n}\right)$. Summing over disjoint events yields

$$
Q^{(n)}=\mathbb{E}\left[\sum \mathbf{1}\left(t_{i_{1}}<\cdots<t_{i_{n}}<0\right) \prod_{j=1}^{n} \mathbf{1}\left(K_{j} \subset \operatorname{Int} V_{i_{j}}\right)\right],
$$


where the sum is taken over all $n$-tuples of points in $\Phi$. First note that only $n$-tuples of distinct points may be considered in this sum and that, from the definition of visible parts in (4) and (C3), the summand in this equation may be written as

$$
\mathbf{1}\left(t_{i_{1}}<\cdots<t_{i_{n}}<0\right) \prod_{j=1}^{n} \mathbf{1}\left(K_{j} \subset\left(x_{i_{j}}+\operatorname{Int} X_{i_{j}}\right)\right) \prod_{\left\{i: t_{i}>t_{i_{j}}\right\}} \mathbf{1}\left(K_{j} \cap\left(x_{i}+X_{i}\right)=\varnothing\right) .
$$

In the simplest case, $n=1$, this amounts to saying that $Q^{(1)}$ is the probability that there exists a leaf, $X_{i}$, such that $K_{1}$ is included in Int $X_{i}$ and is not hit by subsequent leaves. We will now apply the Campbell formula to compute this expectation, and therefore need the following notation. Let $\mathcal{E}:=\mathbb{R}^{2} \times(-\infty, 0] \times \mathcal{F}$. We write $\mathcal{N}^{(n)}($ or $\mathcal{N}$ for $n=1)$ for the space of $\sigma$-finite counting measures on $\mathcal{E}^{n}$. For all $n \geq 1$, we define the point process

$$
\Phi^{(n)}=\sum_{i_{1}, \ldots, i_{n}} \delta_{z_{i_{1}}, \ldots, z_{i_{n}}}
$$

on $\mathcal{E}^{n}$, where the sum is taken over all indices $\left(i_{1}, \ldots, i_{n}\right)$ such that $z_{i_{1}}, \ldots, z_{i_{n}}$ are distinct points of $\Phi$. We define a function $f$ from $\mathcal{E}^{n} \times \mathcal{N}^{(n)}$ to $\mathbb{R}$ such that (10) reads $f\left(\left\{z_{i_{j}}\right\}_{j=1}^{n}, \Phi^{(n)}\right)$. By applying the refined Campbell theorem (see [7]) to compute the expectation in (9), we obtain

$$
Q^{(n)}=\int_{Z \in \mathcal{E}^{n}} \int_{\phi \in \mathcal{N}^{(n)}} f(Z, \phi) \mathbb{P}^{Z}(\mathrm{~d} \phi) \prod_{j=1}^{n} \mu_{\Phi}\left(\mathrm{d} \tilde{z}_{j}\right)
$$

where $Z=\left\{\tilde{z}_{j}\right\}_{j=1}^{n}, \mu_{\Phi}$ is the intensity measure of $\Phi$, and $\mathbb{P}^{Z}$ is the Palm distribution of the process $\Phi^{(n)}$ at $Z$. Applying the generalized Slivnyak theorem (see [26, p. 124]) gives

$$
Q^{(n)}=\int_{Z \in \mathcal{E}^{n}} \mathbb{E}\left[f\left(Z,\left(\Phi+\delta_{\tilde{z}_{1}}+\cdots+\delta_{\tilde{z}_{n}}\right)^{(n)}\right)\right] \prod_{j=1}^{n} \mu_{\Phi}\left(\mathrm{d} \tilde{z}_{j}\right),
$$

where, as usual, $\mathbb{E}$ is the expectation associated to $\Phi$. With $\tilde{z}_{j}=\left(\tilde{x}_{j}, \tilde{t}_{j}, \tilde{X}_{j}\right), j=1, \ldots, n$, and $\tilde{t}_{1}<\cdots<\tilde{t}_{n}<0$, by definition of $f$ we have

$$
\begin{aligned}
f(Z,( & \left.\left.+\delta_{\tilde{z}_{1}}+\cdots+\delta_{\tilde{z}_{n}}\right)^{(n)}\right) \\
= & f\left(Z, \Phi^{(n)}\right) \\
= & \left(\prod_{j=1}^{n} \mathbf{1}\left(K_{j} \subset\left(\tilde{x}_{j}+\operatorname{Int} \tilde{X}_{j}\right)\right)\right)\left(\prod_{j=2}^{n} \mathbf{1}\left(\underline{K}_{j-1} \cap\left(\tilde{x}_{j}+\tilde{X}_{j}\right)=\varnothing\right)\right) \\
& \times \prod_{j=1}^{n-1} \prod_{\left\{i: t_{i} \in\left(\tilde{t}_{j}, \tilde{t}_{j+1}\right]\right\}} \mathbf{1}\left(\underline{K}_{j} \cap\left(x_{i}+X_{i}\right)=\varnothing\right) \prod_{\left\{k: t_{k} \in\left(\tilde{t}_{n}, 0\right]\right\}} \mathbf{1}\left(\underline{K}_{n} \cap\left(x_{k}+X_{k}\right)=\varnothing\right),
\end{aligned}
$$

with $\underline{K}_{j}$ as defined in (7). The expectation in (11) is computed as follows. Since $\Phi$ is a Poisson process, the last line of (12) can be written as a product of independent terms whose expectations can be computed using the fact that, for fixed $s$ and $t, s<t \leq 0$, and for $K$ compact,

$$
\mathbb{P}\left(K \cap\left(x_{i}+X_{i}\right)=\varnothing \text { for all } t_{i} \in(s, t]\right)=\exp ((s-t) \mathrm{E}[v(X \oplus \check{K})])
$$


(see Lemma 1). Next, by integrating with respect to $\mathbf{1}\left(\tilde{t}_{1}<\cdots<\tilde{t}_{n}<0\right) \mathrm{d} \tilde{t}_{1} \cdots \mathrm{d} \tilde{t}_{n}$ and making a change of variable $u_{j}=\tilde{t}_{j}-\tilde{t}_{j+1}, j=1, \ldots, n-1$, we obtain

$$
\begin{aligned}
Q^{(n)}=\prod_{j=1}^{n} \mathrm{E}\left[v\left(X \oplus \underline{\check{K}}_{j}\right)\right]^{-1} \int_{\left(\mathbb{R}^{2} \times \mathcal{F}\right)^{n}} \prod_{j=1}^{n} \mathrm{~d} \tilde{x}_{j} \mathrm{P}\left(\mathrm{d} \tilde{X}_{j}\right) & \left.\left(\prod_{j=1}^{n} \mathbf{1}\left(K_{j} \subset \tilde{(} x_{j}+\operatorname{Int} \tilde{X}_{j}\right)\right)\right) \\
& \times\left(\prod_{j=2}^{n} \mathbf{1}\left(\underline{K}_{j-1} \cap\left(\tilde{x}_{j}+\tilde{X}_{j}\right)=\varnothing\right)\right)
\end{aligned}
$$

The first factor on the right-hand side of this equation is $\left(G^{(n)}\right)^{-1}$, and the integral can be written

$$
\prod_{j=1}^{n}\left(\int_{\mathbb{R}^{2} \times \mathcal{F}} \mathbf{1}\left(K_{j} \subset(\tilde{x}+\operatorname{Int} \tilde{X})\right) \mathbf{1}\left(\underline{K}_{j-1} \cap(\tilde{x}+\tilde{X})=\varnothing\right) \mathrm{d} \tilde{x} \mathrm{P}(\mathrm{d} \tilde{X})\right)
$$

with the convention $\underline{K}_{0}=\varnothing$. Now, for two compact sets, $A$ and $B$, we have

$\int_{\mathbb{R}^{2} \times \mathcal{F}} \mathbf{1}(A \subset(x+\operatorname{Int} X)) \mathbf{1}(B \cap(x+X)=\varnothing) v(\mathrm{~d} x) \mathrm{P}(\mathrm{d} X)=\mathrm{E}\left[v\left((\operatorname{Int} X \ominus \check{A}) \cap(X \oplus \check{B})^{\mathrm{c}}\right)\right]$,

which, together with the last equation, yields $F^{(n)}$ (by 6) and, thus, (8).

For $n=1$ we recover (5), the original result of Matheron. The case $n=2$ was treated in [13]. Note that, from the $Q^{(n)}$, we can compute the probability

$$
\mathbb{P}\left(K_{1} \subset \text { Int } V_{i_{1}}, \ldots, K_{n} \subset \text { Int } V_{i_{n}} \text { for some } i_{1}, \ldots, i_{n} \in \mathbb{N}\right)
$$

and, thus, the probability of $n$ connected compact sets, $K_{1}, \ldots, K_{n}$, avoiding the boundary of the dead leaves tessellation. For $n=2$, for instance, this is

$$
\mathbb{P}\left(\left(K_{1} \cup K_{2}\right) \cap \partial M=\varnothing\right)=Q^{(2)}\left(K_{1}, K_{2}\right)+Q^{(2)}\left(K_{2}, K_{1}\right)+Q^{(1)}\left(K_{1} \cup K_{2}\right) .
$$

Moreover, it is easily seen that if we consider the random field obtained by independently coloring each visible part, then Proposition 5 enables us to compute the finite-dimensional distributions of this field. This is a useful result in the context of image modeling; see [11]. Next, we show that the knowledge of $Q^{(n)}$ for all $n$ characterizes the distribution of $\partial M$ in $\left(\mathcal{F}, \mathscr{B}_{\mathcal{F}}\right)$.

Proposition 6. The distribution of the boundary $\partial M$ is uniquely determined by the functionals $Q^{(n)}, n \in \mathbb{N}$.

Proof. The distribution of $\partial M$ is characterized by its capacity functional, defined, for every compact set $K$, by $\mathbb{P}(F \cap K=\varnothing)$; see [19, p. 30]. Let $K \in \mathcal{K}$, let $\left(r_{n}\right)>0$ be a sequence converging to 0 , and, for each $n$, let $\left(x_{i}^{(n)}\right)_{i=1, \ldots, N_{n}}$ be a finite sequence in $K$ such that $K \subset$ $C_{n}=\bigcup_{i} B\left(x_{i}^{n}, r_{n}\right)$, where $B(x, r)$ is the (closed) ball of radius $r$ centered at $x$. Note that, since each $C_{n}$ is a finite union of connected compact sets, knowledge of the $Q^{(i)}, i \in \mathbb{N}$, uniquely determines $\mathbb{P}\left(C_{n} \cap \partial M=\varnothing\right)$. Now, since $C_{n} \downarrow K$, we have $\mathcal{F}^{C_{n}} \uparrow \mathcal{F}^{K}$ and, thus, $\mathbb{P}\left(C_{n} \cap \partial M=\varnothing\right) \uparrow \mathbb{P}(K \cap \partial M=\varnothing)$. 


\subsection{Typical relief cells}

In this section, we consider the distribution of cells that remain completely visible. This problem was first addressed in [18]; also see [17], [24], and [12].

Definition 4. A cell $V_{i}$ is a relief cell if $\left(x_{i}+X_{i}\right)=V_{i}$. Denote by

$$
N_{r}=\sum_{i} \mathbf{1}\left(V_{i}=\left(x_{i}+X_{i}\right)\right) \delta_{x_{i}, V_{i}}
$$

the point process of relief cells.

As in the proof of Proposition 3, we can show that $N_{r}$ is stationary and mixing. From condition (C3), if $V_{i}=\left(x_{i}+X_{i}\right)$ then Int $V_{i} \neq \varnothing$. It follows that $N_{r}$ is a thinning of $N$, and since $N$ has finite intensity, so has $N_{r}$.

Proposition 7. The typical relief cell distribution is absolutely continuous with respect to $\mathrm{P}$, with Radon-Nikodým derivative $F \mapsto\left(\mu_{r} \mathrm{E}[v(\operatorname{Int} X \oplus \check{F})]\right)^{-1}$, where

$$
\mu_{r}:=\int_{\mathcal{F}} \frac{\mathrm{P}(\mathrm{d} F)}{\mathrm{E}[v(\operatorname{Int} X \oplus \check{F})]}
$$

is the intensity of $N_{r}$.

Remark 4. As a consequence of this proposition, the typical relief cell distribution and the leaf distribution $\mathrm{P}$ are equivalent measures on $\mathcal{I}$. This remark completes the proof of the 'only if' part of Proposition 4.

Proof of Proposition 7. $N_{r}$ is a simple point process with finite intensity. We denote by $\mathbb{P}_{N_{r}}^{0}$ the Palm distribution of $N_{r}$. With $N_{r}=\sum \delta_{x_{i}^{r}}, V_{i}^{r}$, for all $\chi \in \mathcal{I}$ we have

$$
\begin{aligned}
\mathbb{P}_{N_{r}}^{0}\left(V_{0}^{r} \in \chi\right) & =\frac{1}{\mu_{r}} \mathbb{E}\left[\sum_{i} \mathbf{1}\left(V_{i}^{r} \in \chi\right) \mathbf{1}\left(x_{i}^{r} \in[0,1]^{2}\right)\right] \\
& =\frac{1}{\mu_{r}} \mathbb{E}\left[\sum_{i} \mathbf{1}\left(V_{i} \in \chi, x_{i} \in[0,1]^{2},\left(x_{i}+X_{i}\right) \cap \bigcup_{\left\{j: t_{j} \in\left(t_{i}, 0\right]\right\}}\left(x_{j}+\operatorname{Int} X_{j}\right)=\varnothing\right)\right] .
\end{aligned}
$$

From Slivnyak's theorem and Campbell's formula, we obtain

$$
\begin{aligned}
\mathbb{P}_{N_{r}}^{0}\left(V_{0}^{r} \in \chi\right) & =\frac{1}{\mu_{r}} \int_{\mathbb{R}^{2} \times \mathbb{R}_{-} \times \chi} \mathbb{P}\left((x+F) \cap \bigcup_{\left\{j: t_{j} \in(t, 0]\right\}}\left(x_{j}+\operatorname{Int} X_{j}\right)=\varnothing\right) v(\mathrm{~d} x) \mathrm{d} t \mathrm{P}(\mathrm{d} F) \\
& =\frac{1}{\mu_{r}} \int_{\mathbb{R}_{-} \times \chi} \exp (t \mathrm{E}[v(\operatorname{Int} X \oplus \check{F})]) \mathrm{d} t \mathrm{P}(\mathrm{d} F) \\
& =\frac{1}{\mu_{r}} \int_{\chi} \mathrm{E}[v(\operatorname{Int} X \oplus \check{F})]^{-1} \mathrm{P}(\mathrm{d} F),
\end{aligned}
$$

where the second equality follows from Lemma 1 . Taking $\chi=\mathcal{F}^{\prime}$, we also find the claimed formula for the intensity.

As an example, let us compute the area distribution of a typical relief cell: for $\chi_{s}=$ $\left\{F \in \mathcal{F}^{\prime}: v(F)>s\right\}$, we find that

$$
\mathbb{E}_{N_{r}}^{0}\left[v\left(X_{0}^{r}\right)\right]=\frac{1}{\mu_{r}} \int_{\mathcal{F}^{\prime}} v(F) \mathrm{E}[v(\operatorname{Int} X \oplus \check{F})]^{-1} \mathrm{P}(\mathrm{d} F) .
$$


Remark 5. For $d=2$, if $X$ is convex and isotropic a.s., we obtain the original result of Matheron by applying the Steiner formula (see [26, p. 13]) to compute $\mu_{r}$. Let $l(K)$ denote the length of $\partial K$. Then, for $K$ convex we have

$$
\mu_{r}=\mathrm{E}\left[(v(X)+(2 / \pi) l(X) \mathrm{E}[l(X)]+\mathrm{E}[v(X)])^{-1}\right] .
$$

\subsection{Cells intersecting with a line}

We now consider the intersection between the dead leaves model and a fixed line $D$. In this section we take $d \geq 2$ and assume, in addition to (C1)-(C3), that

(C4) $v(\partial X)=0$ a.s. and, for any line $D^{\prime}, D^{\prime} \cap \partial X$ is either empty, finite, or has positive $v_{D^{\prime}}$-measure a.s.

Here $v_{D^{\prime}}$ is the one-dimensional Lebesgue measure on $D^{\prime}$. This assumption holds if $X$ is a.s. a finite union of convex sets, for instance.

We will compute the Palm distribution of the point process $\partial M \cap D$ and, for $X$ convex, prove a result of [18] in the Palm calculus framework.

Lemma 3. $\partial M \cap D$ is a point process on $D$.

Proof. Since $\partial M$ is a.s. a locally finite union of sets $\partial V_{i}$, and since, for all $i, \partial V_{i}$ is included in a finite union of sets $x_{j}+\partial X_{j}$, it is sufficient to show that, for any $j,\left(x_{j}+\partial X_{j}\right) \cap D$ is a.s. a finite or empty set. Let us suppose that this does not hold. Then, by (C4), with positive probability there exists a $j$ such that $v_{D}\left(x_{j}+\partial X_{j}\right)>0$. Thus, $\mathbb{E}\left[v_{D}\left(\bigcup_{j}\left(x_{j}+\partial X_{j}\right)\right)\right]>0$. Without loss of generality, we let $D$ be the first coordinate axis. By Fubini's theorem and translation invariance, we obtain

$$
\mathbb{E}\left[\nu\left\{\bigcup_{j}\left(x_{j}+\partial X_{j}\right)\right\}\right]=\int_{y \in \mathbb{R}^{d-1}} \mathbb{E}\left[v_{D_{y}}\left\{\bigcup_{j}\left(x_{j}+\partial X_{j}\right)\right\}\right] \mathrm{d} y>0,
$$

where, for any $y=\left(y_{2}, \ldots, y_{d}\right), D_{y}$ is the line parallel to $D$ passing through the point $\left(0, y_{2}, \ldots, y_{d}\right)$. Thus, there a.s. exists a $j$ such that $\mathbb{E}\left[v\left(\partial X_{j}\right)\right]>0$, which contradicts $(\mathrm{C} 4)$.

We let $u$ be a unit vector collinear to $D$, denote by $[0, x u]$ the segment $\{\lambda x u, \lambda \in[0,1]\}$, and define, for all $x \geq 0$,

$$
L(x)=\mathbb{P}\left([0, x u] \subset \text { Int } V_{i} \text { for some } i \in \mathbb{N}\right)=Q^{(1)}([0, x u])=\frac{\mathrm{E}[v(\operatorname{Int} X \ominus[0,-x u])]}{\mathrm{E}[v(X \oplus[0,-x u])]},
$$

where $Q^{(1)}$ is as defined above and the last equality follows from (5).

From now on we denote by $N_{\ell}=\sum_{i} \delta_{y_{i}}$ the simple point process defined in Lemma 3 with points in $\mathbb{R}$, and write $\mathbb{P}_{N_{\ell}}$ for its law and $\mathbb{P}_{N_{\ell}}^{0}$ for its associated Palm distribution. We index $N_{\ell}$ such that $\left\{y_{i}\right\}$ is increasing and $y_{0}<0<y_{1}$. The following lemma links the Palm distribution of $N_{\ell}$ to $L$.

Lemma 4. Let $N_{\ell}=\sum_{i} \delta_{y_{i}}$ be the simple stationary point process defined above. Then $L(x)$ is absolutely continuous, has a negative right derivative, $L^{\prime}(0)$, at $x=0$, and is such that, almost everywhere,

$$
\mathbb{P}_{N_{\ell}}^{0}\left(y_{1}>x\right)=\frac{L^{\prime}(x)}{L^{\prime}(0)} .
$$


Proof. Observe that $L(x)=\mathbb{P}_{N_{\ell}}\left(y_{1}>x\right)$ for all nonnegative $x$. Let $\lambda$ be the intensity of $N_{\ell}$. For all $x \geq 0$, the inversion formula (see, e.g. [3, p. 20]) gives

$$
L(x)=\mathbb{P}_{N_{\ell}}\left(y_{1}>x\right)=\lambda \int_{x}^{\infty} \mathbb{P}_{N_{\ell}}^{0}\left(y_{1}>t\right) \mathrm{d} t .
$$

By differentiating, we find that $L^{\prime}(x)=-\lambda \mathbb{P}_{N_{\ell}}^{0}\left(y_{1}>x\right)$. Then, noting that $\mathbb{P}_{N_{\ell}}^{0}\left(y_{1}=0\right)=0$, we obtain the differentiability of $L$ at the origin and find that $L^{\prime}(0)=-\lambda<0$.

We end this section by considering the case of an a.s. convex $X$. First we introduce the geometric covariogram, $\gamma_{X}$, of $X$, defined for $x \geq 0$ by

$$
\gamma_{X}(x):=v(X \cap(x u \oplus X)) .
$$

Note that the covariogram is usually defined on $\mathbb{R}^{d}$, but that here we consider it only on a half-line. Let $p_{u^{\perp}}$ denote the orthogonal projection on the hyperplane orthogonal to $u$, and $v_{u^{\perp}}$ the $(d-1)$-dimensional Lebesgue measure on this hyperplane. If $X$ is convex then $\gamma_{X}$ is a convex function on $\left[0, W_{u}\right)$, where $W_{u}$ is the width of $X$ in the $u$-direction, and is identically 0 outside this interval. Moreover, it is continuously differentiable on $\left[0, W_{u}\right)$, with derivative $\gamma_{X}^{\prime}(x)=-v_{u^{\perp}}\left(p_{u^{\perp}}(X \cap(x u \oplus X))\right) \geq-v_{u^{\perp}}\left(p_{u^{\perp}}(X)\right)$; see [19, p. 86]. From (C1) and $(\mathrm{C} 2)$ we have $\mathrm{E}\left[v_{u^{\perp}}\left(p_{u^{\perp}}(X)\right)\right]<\infty$. Hence, $\mathrm{E}\left[\gamma_{X}\right]$ is absolutely continuous with derivative $\mathrm{E}\left[\gamma_{X}^{\prime}(x)\right]$ at almost every $x \geq 0$. Moreover $\gamma_{X}^{\prime}(x)$ is right continuous at $x=0$ and so is $\mathrm{E}\left[\gamma_{X}^{\prime}(x)\right]$, by dominated convergence; thus, $\mathrm{E}\left[\gamma_{X}(x)\right]$ has right-hand derivative $\mathrm{E}\left[\gamma_{X}^{\prime}(0)\right]=$ $-\mathrm{E}\left[v_{u^{\perp}}\left(p_{u^{\perp}}(X)\right)\right]$ at $x=0$.

Definition 5. The intercept distribution of $X$ (in the $u$-direction) is defined as

$$
F_{X}(x)=\frac{\mathrm{E}\left[\gamma_{X}{ }^{\prime}(x)\right]}{\mathrm{E}\left[\gamma_{X^{\prime}}(0)\right]}, \quad x \geq 0 .
$$

Remark 6. The term 'intercept distribution' is used because $\gamma_{X}^{\prime}(x) / \gamma_{X}^{\prime}(0)$ is the probability distribution of the length of the intersection of $X$ with lines, uniformly distributed among those hitting $X$, having direction $u$; see [24].

Proposition 8. Let $M$ be a dead leaves model associated with a random closed set, $X$, which is convex with intercept distribution $F_{X}$ a.s., and let $\mathbb{P}_{N_{\ell}}^{0}$ and $y_{1}$ be defined as above. Then, for all $x \geq 0$,

$$
\int_{x}^{\infty} \mathbb{P}_{N_{\ell}}^{0}\left(y_{1}>t\right) \mathrm{d} t=\frac{1}{2}(1+K x)^{-1} \int_{x}^{\infty} F_{X}(t) \mathrm{d} t,
$$

where $K=-\mathrm{E}\left[\gamma_{X}^{\prime}(0)\right] / \mathrm{E}\left[\gamma_{X}(0)\right]$.

Proof. It can be shown that, when $X$ is convex, $v(X \ominus[0,-x u])=\gamma_{X}(x)$ and

$$
v(X \oplus[0,-x u])=\gamma_{X}(0)+x v_{u^{\perp}}\left(p_{u^{\perp}}(X)\right) .
$$

Since $v_{u^{\perp}}\left(p_{u^{\perp}}(X)\right)=-\mathrm{E}\left[\gamma_{X}^{\prime}(0)\right],(13)$ yields

$$
L(x)=\frac{\mathrm{E}\left[\gamma_{X}(x)\right]}{\mathrm{E}\left[\gamma_{X}(0)\right]-x \mathrm{E}\left[\gamma_{X}^{\prime}(0)\right]},
$$

and the result then follows easily from (14) and (15). 
Let us finally note that $\mathbb{P}_{N_{\ell}}^{0}\left(y_{1}>x\right)$ may be seen (as in Section 2.2) as the length distribution of the 'typical cell' of the tessellation $D \cap M:=\sum_{i} \mathbf{1}\left(V_{i} \cap D \neq \varnothing\right) \delta_{V_{i} \cap D}$, and thus as the intercept distribution of the typical cell of $M$ (which is not convex). Note also that, by setting $x=0$ in (16), we obtain

$$
\mathbb{E}_{N_{\ell}}^{0}\left[y_{1}\right]=\frac{1}{2} \int_{0}^{\infty} F_{X}(t) \mathrm{d} t,
$$

which show (see Remark 6) that, for $X$ convex, the mean intercept in any direction is divided by two as a result of occlusion.

\section{Conclusion}

Various generalizations of this model are possible. Inhomogeneous point processes could be considered, or the independence assumption between time and objects could be broken (see [12]), enabling perspective laws to be taken into account. In the homogeneous and independent case, many open problems remain, in particular to do with computing typical cell properties, given the distribution of the leaf $X$. The computation of the mean perimeter and area of typical cells, as performed in [5] for the connected components of visible parts, is an interesting direction for further work.

\section{References}

[1] Alvarez, L., Gousseau, Y. And Morel, J.-M. (1999). The size of objects in natural and artificial images. Adv. Imaging Electron Phys. 111, 167-242.

[2] Ambartzumian, R. V. (1974). Convex polygons and random tessellations. In Stochastic Geometry, eds E. F. Harding and D. G. Kendall, John Wiley, New York, pp. 176-191.

[3] Baccelli, F. and Bremaud, P. (2002). Elements of Queuing Theory (Appl. Math. 26), 2nd edn. Springer, Berlin.

[4] Cowan, R. (1980). Properties of ergodic random mosaic processes. Math. Nachr. 97, 89-102.

[5] Cowan, R. And Tsang, A. K. L. (1994). The falling-leaves mosaic and its equilibrium properties. Adv. Appl. Prob. 26, 54-62.

[6] Cowan, R. and Tsang, A. K. L. (1995). Random mosaics with cells of general topology. Res. Rep. 89, University of Hong Kong.

[7] Daley, D. J. and Vere-Jones, D. (1988). An Introduction to the Theory of Point Processes. Springer, New York.

[8] Gille, W. (2002). The set covariance of a dead leaves model. Adv. Appl. Prob. 34, 11-20.

[9] Goffman, C. And Pedrick, G. (1975). A proof of the homeomorphism of Lebesgue-Stieltjes measure with Lebesgue measure. Proc. Amer. Math. Soc. 52, 196-198.

[10] Gousseau, Y. (2002). Texture synthesis through level sets. In Texture 2002 (Proc. 2nd Internat. Workshop Texture Anal. Synthesis, Copenhagen, 2002), 5pp. Available at http://www.cee.hw.ac.uk/ texture2002/ index.html\#ab044.

[11] Gousseau, Y. And RouefF, F. (2003). The dead leaves model: general results and limits at small scales. Preprint. Available at http://arxiv.org/abs/math.PR/0312035.

[12] Jeulin, D. (1989). Morphological modeling of images by sequential random functions. Advances in mathematical morphology. Signal Process. 16, 403-431.

[13] Jeulin, D. (1996). Dead leaves models: from space tessellation to random functions. In Proc. Internat. Symp. Adv. Theory Applications Random Sets (Fontainebleau, 1996), ed. D. Jeulin, World Scientific, River Edge, NJ, pp. $137-156$.

[14] Jeulin, D., Villalobos, I. T. and Dubus, A. (1995). Morphological analysis of $\mathrm{UO}_{2}$ powder using a dead leaves model. Microsc. Microanal. Microstruct. 6, 371-384.

[15] Kendall, W. S. And Thonnes, E. (1999). Perfect simulation in stochastic geometry. Pattern Recognition 32, $1569-1586$.

[16] Lee, A. B., Mumford, D. And Huang, J. (2001). Occlusion models for natural images: a statistical study of a scale-invariant dead leaves model. Internat. J. Computer Vision 41, 35-59.

[17] Månsson, M. and Rudemo, M. (2002). Random patterns of nonoverlapping convex grains. Adv. Appl. Prob. 34, 718-738.

[18] Matheron, G. (1968). Modèle séquentiel de partition aléatoire. Tech. Rep., Centre de Morphologie Mathématique, Fontainebleau. 
[19] Matheron, G. (1975). Random Sets and Integral Geometry. John Wiley, Chichester.

[20] Mecke, J. (1980). Palm methods for stationary random mosaics. In Combinatorial Principles in Stochastic Geometry, ed. R. V. Ambartzumian, Armenian Academy of Sciences, Erevan, pp. 124-132.

[21] Møller, J. (1989). Random tessellations in $\mathbb{R}^{d}$. Adv. Appl. Prob. 21, 37-73.

[22] Okabe, A., Boots, B., Sugihara, K. and Chiu, S. N. (2000). Spatial Tessellations: Concepts and Applications of Voronoi Diagrams, 2nd edn. John Wiley, Chichester.

[23] Ruderman, D. L. (1997). Origins of scaling in natural images. Vision Res. 37, 3385-3398.

[24] Serra, J. (1982). Image Analysis and Mathematical Morphology. Academic Press, London.

[25] Stoyan, D. (1986). On generalized planar tessellations. Math. Nachr. 128, 215-219.

[26] Stoyan, D., Kendall, W. S. And Mecke, J. (1995). Stochastic Geometry and its Applications, 2nd edn. John Wiley, Chichester.

[27] Weiss, V. AND ZäHLE, M. (1988). Geometric measures for random curved mosaics of $\mathbf{R}^{d}$. Math. Nachr. 138, 313-326. 